\title{
The influence of Titan on Saturn kilometric radiation
}

\author{
J. D. Menietti ${ }^{1}$, S.-Y. Ye ${ }^{1}$, C. W. Piker ${ }^{1}$, and B. Cecconi ${ }^{2}$ \\ ${ }^{1}$ University of Iowa, Department of Physics and Astronomy, 210 Van Allen Hall, Iowa City, IA 52242 USA \\ ${ }^{2}$ Observatoire de Paris, Meudon 92195, France
}

Received: 9 June 2009 - Revised: 21 September 2009 - Accepted: 22 January 2010 - Published: 3 February 2010

\begin{abstract}
Previous studies have shown that the occurrence probability of Saturn Kilometric Radiation (SKR) appears to be influenced by the local time of Titan. Using a more extensive set of data than the original study, we confirm the correlation of higher occurrence probability of SKR when Titan is located near local midnight. In addition, the direction finding capability of the Cassini Radio Plasma Wave instrument (RPWS) is used to determine if this radio emission emanates from particular source regions. We find that most source regions of SKR are located in the mid-morning sector of local time even when Titan is located near midnight. However, some emission does appear to have a source in the Saturnian nightside, consistent with electron precipitation from field lines that have recently mapped to near Titan.
\end{abstract}

Keywords. Magnetospheric physics (Planetary magnetospheres; Plasma waves and instabilities) - Space plasma physics (Wave-particle interactions)

\section{Introduction}

Voyager observations of Saturn kilometric radiation revealed emission in the frequency range from a few $\mathrm{kHz}$ to about $1.3 \mathrm{MHz}$, with a peak intensity near $200 \mathrm{kHz}$ (cf. Gurnett et al., 1981; Warwick et al., 1981, 1982; Kaiser et al., 1984). SKR shows predominantly right hand polarization and wavelengths in the kilometer band, similar to terrestrial auroral kilometric radiation. A peculiar feature of the Saturn SKR is that it appears to display clock-like periodicity with more intense emission occurring when a particular Saturn longitude is near the sub-solar point (Gurnett et al., 1981; Warwick et al., 1981). Kaiser et al. (1981), Kaiser and Desch (1982), and Lecacheux and Genova (1983) have reported that the radio

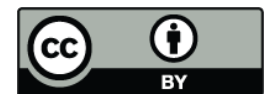

Correspondence to: J. D. Menietti (john-menietti@uiowa.edu) emission sources are in both hemispheres, with the strongest being the Northern Hemisphere. Using the original Saturn longitude system, SLS, the northern source appears to be confined to magnetic field lines with footprints in the range $70^{\circ}<$ latitude $<80^{\circ}$ and $100^{\circ}<\mathrm{SLS}<130^{\circ}$, and the sources are strongest in the local time range 10:00 $\mathrm{h}<\mathrm{LT}<12: 00 \mathrm{~h}$. The Southern Hemisphere source appears to be in the range $-60^{\circ}<$ latitude $<-85^{\circ}$ and $300^{\circ}$ to $75^{\circ} \mathrm{SLS}$, and strongest in the broader range 07:00 $\mathrm{h}<\mathrm{LT}<16: 00 \mathrm{~h}$. These observations have never been completely explained, and due to the apparent drifting of the SLS system, it is important to determine how these observations may have changed.

Cecconi et al. (2006) and Lamy et al. (2008a) have summarized the results of direction finding and polarization measurements of SKR using the capabilities of the Radio and Plasma Wave Instrument (RPWS) on board Cassini. The latter authors find that their results are consistent with SKR sources at or close to the local electron cyclotron frequency in the local time (LT) sector 09:00 h to $12: 00 \mathrm{~h}$, and latitudes $\geq 70^{\circ}$, with emission beamed along hollow cones centered on the local magnetic field vector. Lamy et al. (2008a) have reported that SKR sources are observed at all local times, with more intense radio emissions in the morning to noon sector. Farrell et al. (2005) first reported an SKR radio source from an active region in the night-side inner magnetosphere, and nightside emission has recently been reported by Lamy et al. (2008b) and Cecconi et al. (2009). This is in contrast to many previous reports that the SKR source region is located in the morning sector at high latitude. More recently, Cecconi et al. (2009) present a comprehensive goniopolarimetric (direction-finding) study of SKR which provides beaming angle, invariant latitude, and local time of the SKR sources. The emission is shown to be primarily in the right-hand extraordinary (R-X) mode, with marginal left-hand (L-O) mode emission. The footprints of active magnetic field lines lie in the $\sim 70^{\circ}$ to $\sim 80^{\circ}$ northern and southern latitudinal range and in the $04: 00 \mathrm{~h}$ to $16: 00 \mathrm{~h}$ local time range, matching

Published by Copernicus Publications on behalf of the European Geosciences Union. 
observations of the UV and IR aurorae, and including some nightside sources, but with no discussion of the relationship to the local time of Titan.

The influence of Titan on the occurrence probability (OP) of Saturn Kilometric Radiation (SKR) has been reported by Menietti et al. (2007). These authors suggested that Titan's presence in the magnetotail may contribute to the efficiency of reconnection and/or may increase mass loading onto reconnected field lines that map to the auroral region and active SKR generation sites. Titan is a significant source of pick-up ions and mass loading (cf. Mitchell et al., 2005; Szego et al., 2005), and is believed to have Alfven wings and an extensive wake (cf. Neubauer et al., 2006; Ma et al., 2006). Modolo and Chanteur (2008) indicate mass loss rates near Titan are $\sim 6 \times 10^{25}$ ions/s. Neutral imaging from the Magnetospheric Imaging Instrument (MIMI) and Ion Neutral Camera (INCA) show distinct sources of plasma in the magnetotail associated with Titan. Mitchell et al. (2005) state that the association of SKR with ion enhancements is an indication of Earthlike substorm activity at Saturn. The occurrence probability of SKR associated with the local time of Titan is consistent with the observations of Mitchell et al. (2005) and suggests substorm-like events with rapid and large increases in SKR even before the neutral ion cloud rotates to the dayside. Jackman et al. (2009) have shown the association of SKR radio emissions and plasmoid-like structures indicative of Saturn substorms. However, Menietti et al. (2007) found no significant association of SKR emission with the orbital location of Enceladus, despite the known significant source of water ions associated with this moon (cf. Porco et al., 2006). One possible explanation for this lack of direct association is the fact that SKR source magnetic field lines are located at high L-shells as determined by Lamy et al. (2008a), while the Enceladus orbit is near $4 R_{\mathrm{S}}\left(R_{\mathrm{S}}=\right.$ Saturn radius $)$.

Kurth et al. (2005) and, Lamy et al. (2009) have reported observations of SKR that correlate with UV observations of the Hubble Space Telescope. They report that SKR can be associated with bright auroral features just as terrestrial auroral kilometric radiation. In addition, Wang et al. (2008) examine the possible association of narrowband radio emissions observed by Cassini RPWS with a revolving plasma cloud detected by the MIMI/INCA (Magnetospheric Imaging/Ion and Neutral Camera) instrument onboard Cassini. Narrowband emission, with a source region distinct from SKR, is nevertheless known to correlate with SKR enhancements (cf. Ye et al., 2009). Narrowband emission is believed to have a source mechanism that involves wave mode conversion (similar to continuum emission at Earth) and distinct from the cyclotron maser instability (the source of SKR, cf. Menietti et al., 2009).

Recently, Russell et al. (2008) have shown that observations of probable magnetic reconnection at Saturn occur when Titan is very near a local time of midnight on 4 of 7 occurrences. These authors attribute the influence of plasma pickup ions and the stretching of magnetic field lines in the vicinity of Titan to this observation, and make the case for Saturnian substorms. Jackman et al. (2007, 2008, 2009) also present evidence of reconnection and plasmoid formation that substorms occur in the magnetotail of Saturn. Zaitsev et al. (2010) discuss the acceleration of electrons in Titan's ionosphere, and propose that these electrons might be an occasional source of Saturn kilometric radiation (SKR). The ionospheric electron acceleration mechanism involves the induced electric fields due to Titan's motion through the Saturnian magnetosphere, and the parallel component of the charge separation electric field. The overall estimate of the electron energy is $\sim 5 \mathrm{keV}$ and this energy appears also to be sufficient to explain the UV flux density observed in the Titan atmosphere. Zaitsev et al. (2010) provide a possible explanation for the apparent association of Titan with SKR occurrence probability (Menietti et al., 2007; Khodachenko et al., 2008). In addition, Winglee et al. (2009) have performed three-dimensional multifluid/multiscale modeling of the interaction of Titan and its ion tail with the Saturn magnetosphere. These authors show that during conditions of antiparallel IMF the interchange instability drives outward fingers of cold, dense plasma from the inner magnetosphere. Titan interacts with these fingers causing enhanced outflow and large changes in the orientation of the Titan's ion tail. The important point made by the authors is that the ion tail modifies the position of the inner boundary of the plasma sheet, which in turn may influence substorm activity and aurorae. Winglee et al. (2009) state "Titan's ion tail acts as a barrier to the magnetospheric flows and creates tenuous regions of hot plasma which aide in the creation of conditions that favor SKR".

In this paper we re-examine the occurrence probability of SKR emissions using a much larger database than the original study of Menietti et al. (2007). We find the general conclusions of that study are confirmed. In addition we isolate emission associated with Titan and determine the direction to the source. We find some of this emission does appear to result from field lines that map to the nightside of Saturn.

\section{Instrumentation}

The Cassini Radio and Plasma Wave Science (RPWS) instrument measures oscillating electric fields over the frequency range $1 \mathrm{~Hz}$ to $16 \mathrm{MHz}$ and magnetic fields in the range $1 \mathrm{~Hz}$ to $12 \mathrm{kHz}$ (cf. Gurnett et al., 2004). The instrument uses 3 nearly orthogonal electric field antennas and 3 orthogonal magnetic search coil antennas, providing a direction-finding capability. There are 5 receiver systems: the high frequency receiver (HFR) covering $3.5 \mathrm{kHz}$ to $16 \mathrm{MHz}$; the medium frequency receiver (MFR) covering $24 \mathrm{~Hz}$ to $12 \mathrm{kHz}$; a low frequency receiver covering $1 \mathrm{~Hz}$ to $2.5 \mathrm{kHz}$, and a high resolution wideband receiver that covers two frequency bands, $60 \mathrm{~Hz}$ to $10.5 \mathrm{kHz}$, and $800 \mathrm{~Hz}$ to $74 \mathrm{kHz}$. The data presented in this study utilize the HFR exclusively. 


\section{Observations}

We proceed in a fashion similar to our previous study by determining the occurrence probability of radio emission in bins of Titan orbital phase versus spacecraft SKR longitude. Orbital phase is defined relative to superior conjunction with the spacecraft. The SKR longitude system used in this study has been described in some detail in Kurth et al. (2007, 2008), and is a variable system, locked relative to the period of the Saturn kilometric radiation.

Instead of examining the radio emission in separate frequency bins as in the previous study, we integrate the power spectral density (normalized to $100 R_{\mathrm{S}}$ ) over the entire nominal range of SKR emission, $100 \mathrm{kHz}$ to $900 \mathrm{kHz}$. The lower cutoff of SKR is chosen to avoid the numerous observations of narrowband emission (see Introduction) at frequencies typically in the range $5 \mathrm{kHz}-40 \mathrm{kHz}$ (cf. Louarn et al., 2007; Lamy et al., 2008a; Ye et al., 2009). We evaluate the mean intensity over successive 1-min time intervals for the specified frequency range. A threshold power level is determined for each 10-day period as 1.5 times the average mean value. Menietti et al. (2007) analyzed data obtained during the period 18 June 2004 to 29 June 2006. In this present study we extend the time period to 1 January 2008. In addition the dataset utilized has a higher frequency resolution than that used in the previous study.

During this time period the orbit varied considerably. Prior to about day 340, 2004, Cassini orbited with an inclination of about -17 degrees. From this date to about day 109, 2005 , the inclination was generally less than about -5 degrees. Between day 109 and day 245, 2005, the inclination varied from -10 to -15 degrees, but after day 250,2005 , the inclination was near zero. The orbit has also been precessing clockwise in local time, and after day 300, 2005, the orbit precessed rather rapidly from the morning sector through the post-midnight sector. This condition persisted until about day 230, 2006, when the orbit plane was modified to higher inclinations for the rest of the sampling period.

Figure 1 is a plot of the orbits of the Cassini spacecraft in the $x-y$ plane of Saturn solar equatorial (SSQ) coordinates during the data sampling period. In this coordinate system $+\mathrm{x}$ is toward the sun in the Saturn-sun plane and $+\mathrm{z}$ is along the northward spin axis of Saturn, and $\hat{y}=\hat{z} x \hat{x}$. While the orbital coverage is not a homogeneous sampling of the orbital space, the spacecraft covers all local times, latitudes ranging from $-50^{\circ}$ to $+60^{\circ}$, and a radial distance in the range $3 R_{\mathrm{S}}<r<100 R_{\mathrm{S}}$. Figure 2 is a summary plot of the results in the frequency range $100 \mathrm{kHz}<f<900 \mathrm{kHz}$ measured by the RPWS for the data period shown. We plot Titan local time versus the SKR longitude of the sub-solar point (which we call Saturn heliocentric longitude or SHL) with the OP color-coded. The bin size is $6^{\circ} \times 6^{\circ}$. The occurrence probability (OP) is defined as the ratio of the number of times a signal was detected above the threshold level (1.5 times the mean value) divided by the total number of signals

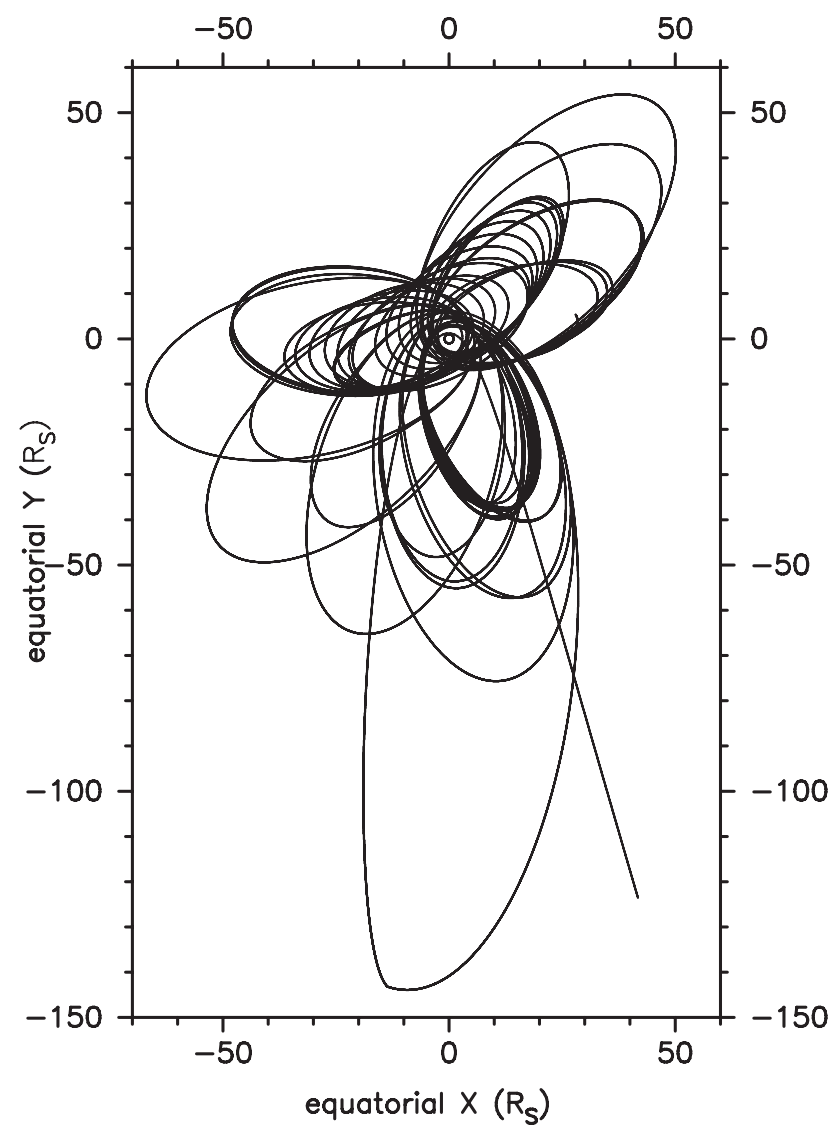

Fig. 1. Orbits of the Cassini spacecraft in the equatorial $x-y$ plane during the data sampling period. The spacecraft covers all local times, latitudes ranging from $-50^{\circ}$ to $+60^{\circ}$, and a radial distance in the range $3 R_{\mathrm{S}}<r<100 R_{\mathrm{S}}$.

above the background level within the $6^{\circ} \times 6^{\circ}$ bin and the frequency range. Note how the $\mathrm{OP}$ is enhanced for the range of 21:00 $\mathrm{h}<\mathrm{LT}<05: 00 \mathrm{~h}$, centered near 02:00 h, and with a smaller peak centered near $9 \mathrm{~h}$. The data also indicate a range of SHL in which the occurrence probability appears to be enhanced (centered near $\sim 110^{\circ}$ ), i.e., a radio emission active zone. This zone in SHL reflects the clock-like periodicity of the Saturn emission, as reported in the past (cf. Gurnett et al., 1981; Warwick et al., 1981). In Fig. 3 we plot the occurrence probability versus Titan local time but we have averaged the $\mathrm{OP}$ over the range, $42^{\circ}<\mathrm{SHL}<180^{\circ}$. This range was chosen to be centered near SHL $\sim 110^{\circ}$ including the active zone and minimizing the influence of the much weaker emission outside this region. Representative error bars of length $2 \sigma$ ( $\sigma=$ standard deviation) are included for a number of the points. This error bar only reflects the range of occurrence probabilities over the chosen range of SHL. In order to understand the secondary peak near 09:00 h LT it is important to note that the orbits of Cassini during much of the data-collecting period were in the morning sector (with the line of apsides approximately centered near 08:00-09:00 h of 


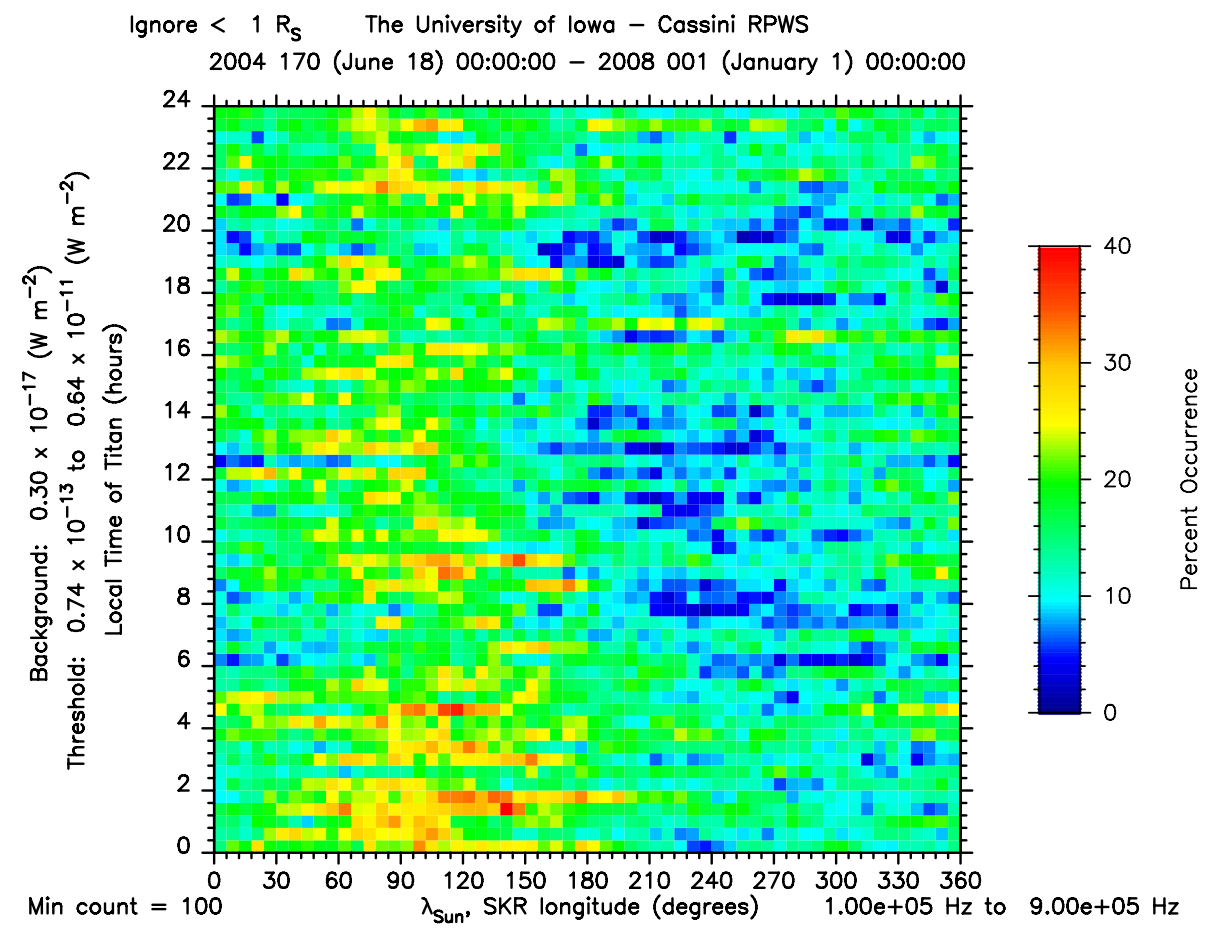

Fig. 2. Summary plot in the frequency range $100 \mathrm{kHz}<f<900 \mathrm{kHz}$, for data obtained over the period from 18 June 2004 to $1 \mathrm{January} 2008$. The local time of Titan is plotted versus SKR longitude with occurrence probability of SKR color-coded according to the color bar at right.

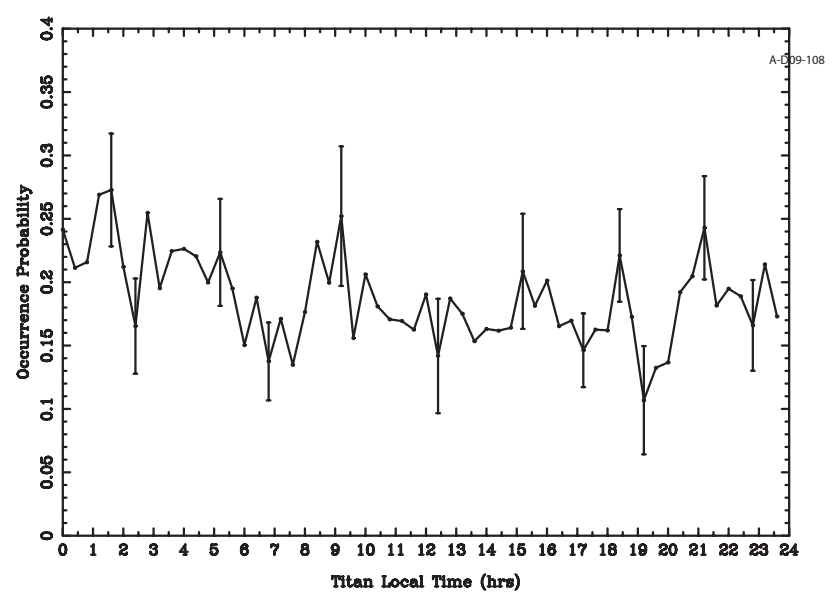

Fig. 3. The occurrence probability versus Titan local time with the $\mathrm{OP}$ averaged over the range, $42^{\circ}<\mathrm{SHL}<180^{\circ}$. The representative error bars on a number of points have a length of $2 \sigma$.

local time), precessing in the later months into and through the post-midnight sector. This is seen in Fig. 1. We also note that most sources of SKR are believed to be in the morning sector (cf. Lamy et al., 2008a). When Titan is in the range 08:00-10:00 $\mathrm{h}$ of local time, it is generally in-line with the Cassini spacecraft and Saturn. Thus Cassini may observe an enhancement of SKR emission because some radio emission from gyroresonant sources near Saturn along the Titan flux tube is beamed in the Titan meridian plane as discussed in Menietti et al. (2007).

\section{Direction Finding (DF) of sources}

One of the capabilities of the RPWS is direction finding from measured signal phase differences between the antennas (cf. Cecconi and Zarka, 2005). By using antenna auto and cross correlations of the antennas, the direction of arrival, Poynting flux, and polarization of an assumed plane wave (all four Stokes parameters) can be obtained. The instrument operates in either a 2-antenna (dipole-monopole) or a 3-antenna mode. For the 2-antenna mode the assumption of circular polarization and no linear polarization is made $(Q=U=0)$ to obtain all the Stokes parameters. Regardless of mode, the instrument is limited to observations when the direction to the source (assumed to be a point source of angular extent $<5^{\circ}$ as shown by Cecconi, 2007) is not near the antenna plane. For the 2-antenna mode Ye et al. (2009) point out that the instrument is limited to observations of latitude less than about $30^{\circ}$, before errors become excessive (this restriction can be removed for the 3-antennas mode). It has been shown (Fischer et al., 2009) that these "errors" come from elliptical polarization of the SKR waves, so the circular polarization assumption is not valid. For a freely propagating wave of strong circular polarization and with amplitude $\geq 23 \mathrm{~dB}$, the direction to the source can be obtained to within about $2^{\circ}$ for 


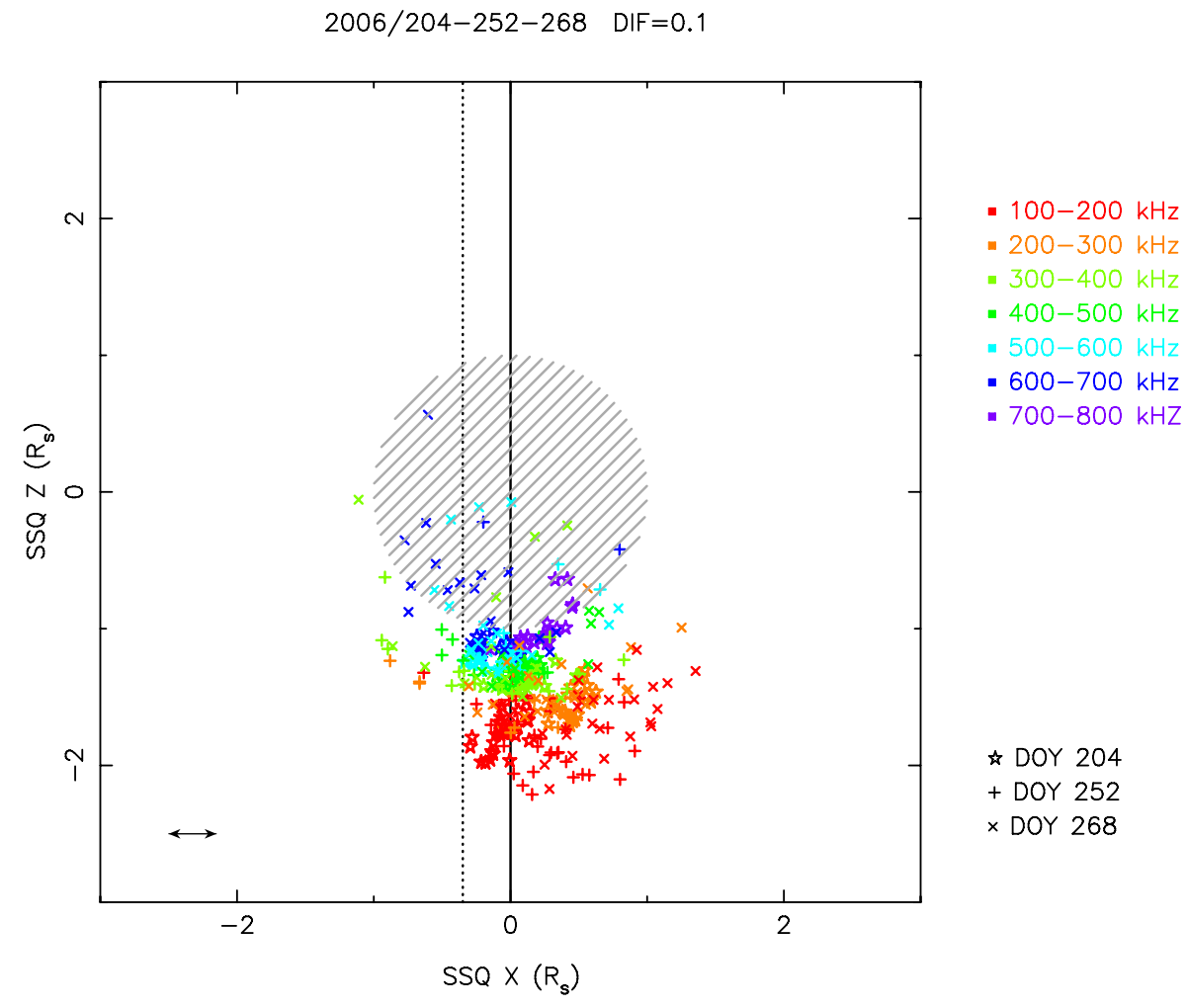

Fig. 4a. Results of direction finding in the SSQ x-z plane for time periods during 3 passes for which all four constraints above were satisfied. These include days 204, 252, and 268 of 2006. The time intervals that satisfied the criteria are listed as well as the actual time of direction finding and the frequency range. The symbol code corresponds to different days, and the color code defines the frequency range.

measurements obtained within about $10 R_{\mathrm{S}}$ (cf. Cecconi and Zarka, 2005; Ye et al., 2009; B. Cecconi, private communication).

We selectively sort the bins of Fig. 2 for occurrence probability, $\mathrm{OP}>0.25(25 \%)$, and extract the data times. For specific intervals within these time periods we perform DF using the RPWS 2-antenna mode information. For the screened time periods, no data were obtained in the 3-antenna mode. The SKR is assumed to be gyroresonant emission from sources along Saturn magnetic field lines (cf. Zarka, 1998; Lamy et al., 2008a). DF is performed at a specific frequency by obtaining the direction angles $(\theta, \phi)$ to the source measured in spacecraft coordinates, using a rotation matrix to convert these directions to Saturn solar equatorial (SSQ) coordinates, then following this direction to the point of intersection with a gyroresonant surface at or near the observed frequency. To perform this direction-finding survey we required the following selection criteria:

1. $\mathrm{OP} \geq 0.25(25 \%)$

2. Titan lies in the range of local times 21:00h $<$ LT $<05: 00 \mathrm{~h}$

3. Cassini radial distance $<10 R_{\mathrm{S}}$
4. Cassini local time (lt) in the ranges 05:00 $<\mathrm{lt}<07: 00$ OR $17: 00<1 \mathrm{t}<19: 00$

It is also important to know the angle, $\beta$, between the direction to the center of Saturn and the antenna plane. Best direction finding results in the 2 -antenna mode are obtained for $20^{\circ}<\beta<60^{\circ}$. In addition, the latitude of the spacecraft should be $<30^{\circ}$ for reliable direction finding. This latter condition is satisfied for all of the source regions we consider in this report. Criterion 4 was applied in order to increase the ability to distinguish between dayside and nightside sources. In other words, DF for Cassini locations at other positions might be ambiguous if the line of sight from the spacecraft intercepts both dayside and nightside sources. Criteria 1-4 are stringent and result in only 3 passes of limited time intervals as listed in Table 1.

In Fig. 4a, b we display the results of direction finding in Saturn solar equatorial (SSQ) coordinates for time periods during 3 passes for which all four constraints above were satisfied. These are days 204, 252, and 268 of 2006. In Table 1 the time intervals that satisfied the criteria are listed as well as the actual time of DF and the frequency range. A different symbol represents each different day as depicted. The color code defines the frequency range as displayed. For a magnetic field closely aligned with the rotation axis, as is the 


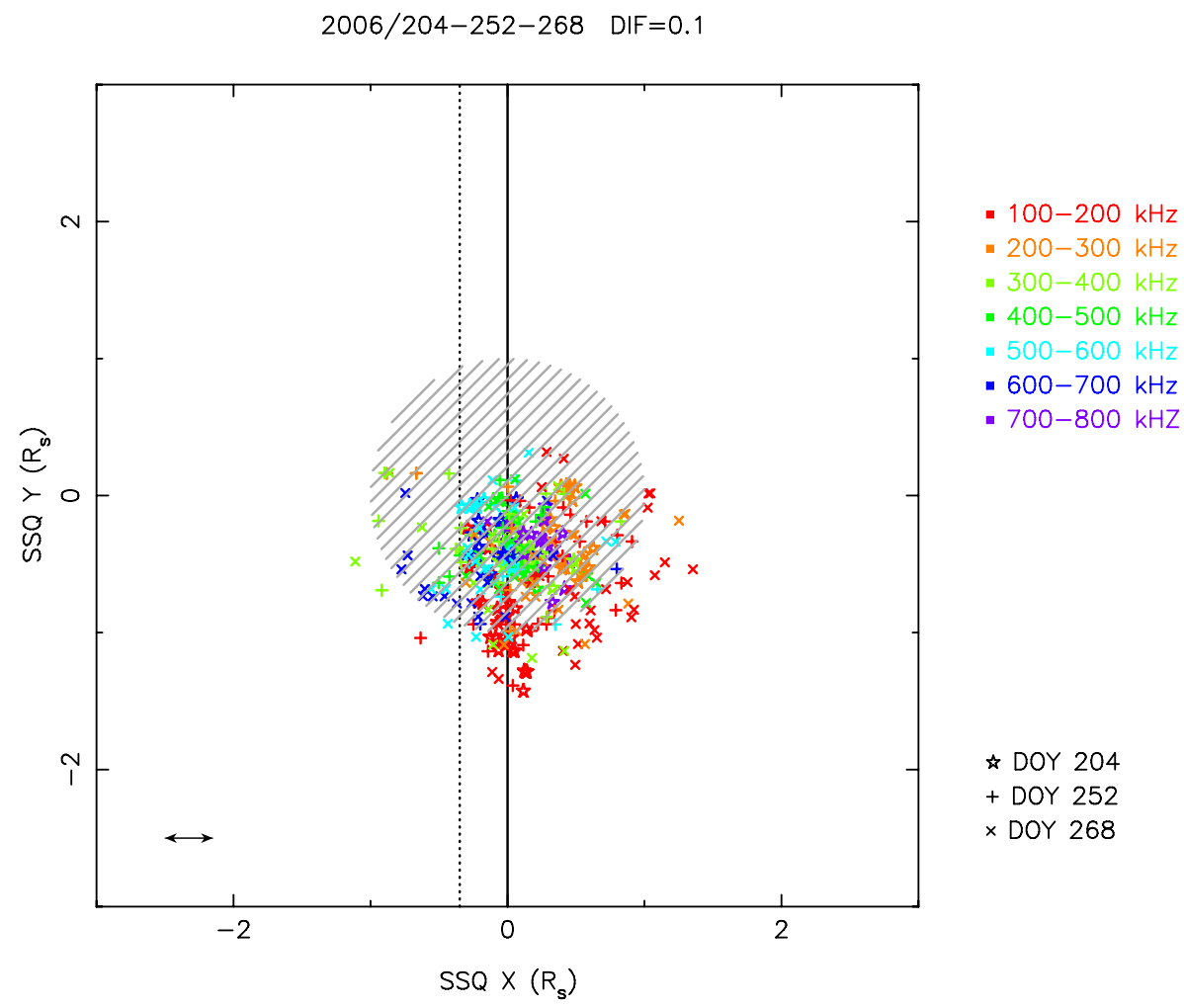

Fig. 4b. Same as Fig. 4a, but now in the $x-y$ plane.

Table 1. Summary of DF events.

\begin{tabular}{lcccccc}
\hline Event day & Appx. evnt time & DF time & $\begin{array}{c}\Delta t \\
(\mathrm{sc})\end{array}$ & $\begin{array}{c}\Delta f \\
(\mathrm{kHz})\end{array}$ & $\begin{array}{c}r \\
\left(R_{S}\right)\end{array}$ & $\begin{array}{c}\sim \beta \\
(\mathrm{deg} .)\end{array}$ \\
\hline $2005 / 122^{\mathrm{a}}$ & $14: 59-15: 23$ & $15: 16: 54$ & 96 & $101-444$ & 7.6 & 50 \\
$2005 / 213^{\mathrm{a}}$ & $14: 13-14: 35$ & $14: 14: 12$ & 80 & $104-619$ & 9.9 & 55 \\
$2005 / 231^{\mathrm{a}}$ & $23: 03-23: 59$ & $23: 11: 22$ & 80 & $104-769$ & 8.4 & 62 \\
$2005 / 232^{\mathrm{a}}$ & $00: 11-03: 25$ & $02: 59: 06$ & 64 & $104-644$ & 6.5 & 58 \\
& $21: 13-21: 23$ & & & & & \\
$2006 / 204$ & $11: 15-11: 19$ & & & & & \\
& $13: 51-15: 59$ & $15: 48: 15$ & 64 & $104-794$ & 5.7 & 30 \\
$2006 / 252$ & $06: 03-06: 45$ & & & & & \\
& $07: 31-09: 05$ & $08: 49: 08$ & 64 & $104-644$ & 7.0 & 45 \\
$2006 / 268$ & $09: 43-09: 59$ & $09: 44: 44$ & 80 & $104-694$ & 7.3 & 60 \\
\hline
\end{tabular}

a Criteria 1-3

case with Saturn, SSQ is an appropriate coordinate system in which to display our results.

Points are included on Fig. 4a, b if the direction from the spacecraft to the source at frequency $f$ intersects the gyrosurface, $f_{\mathrm{c}}$, satisfying the constraint, $\operatorname{dif}=\left|f-f_{\mathrm{c}}\right| / f<0.1$. The values of the magnetic field used to determine $\mathrm{f}_{c}$ were calculated using a simple dipole with moment $=.21184$ gauss- $r_{s}^{3}$. The arrow in the lower left corner represents an error bar. It indicates the projected arc length of $2^{\circ}$ from a distance of
$10 R_{\mathrm{S}}$, which represents the maximum error expected for direction finding under nominal conditions. These conditions include spacecraft distances $<10 R_{\mathrm{S}}$ and $\beta<60^{\circ}$. We have also drawn a dashed line parallel to the y-axis at $x_{o}=-.349 R_{\mathrm{S}}$ a position from the origin equal to the length of the arrow. Most of the points lie in the dayside morning sector. There are a number of points, particularly those at higher frequency, that lie on the nightside. Most of those for negative $x$ lie to the right of the dashed line, indicating they may be within 


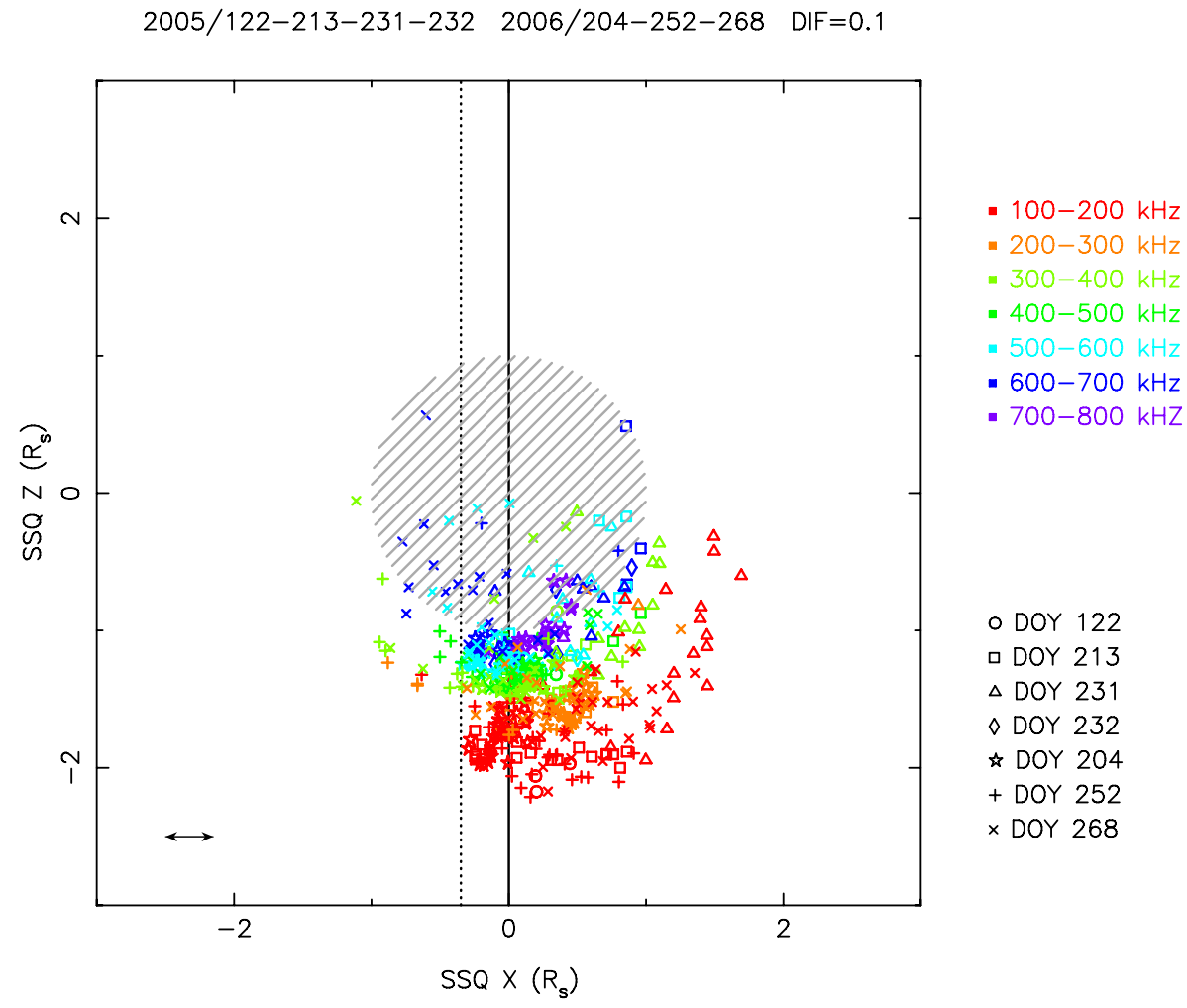

Fig. 5a. Results of direction finding in the SSQ x-z plane for time periods with criterion 4 removed, thus expanding the days to include passes on days $122,213,231$, and 232 of 2005 .

Table 2. Sorting of DF results for Fig. 4.

\begin{tabular}{ccccc}
\hline & $f<300 \mathrm{kHz}$ & $300-600 \mathrm{kHz}$ & $f>600 \mathrm{kHz}$ & Total $(\%)$ \\
\hline$x<x_{o}$ & 1 & 18 & 7 & 7.8 \\
$x_{0}<x<0$ & 33 & 58 & 0 & 27.3 \\
$x>0$ & 107 & 65 & 44 & 64.9 \\
Total $(\%)$ & 42.3 & 42.3 & 15.3 & \\
\hline
\end{tabular}

the error limit for sources on the dayside. In Table 2 we have sorted the data of Fig. 4a, b by frequency range and by location. The entries in the first three columns and rows are number of points. The table makes it clearer that the majority of the points $(64.9 \%)$ lie at $x>0$, but a significant number lie on the nightside, with $7.8 \%$, mostly at higher frequencies, for $x<x_{o}$.

In order to expand the dataset we relaxed the constraints by omitting criterion 4 . The four days with asterisks in Table 1 designate additional periods thus added. In Fig. 5a, b we present the DF including these additional times to compare with Fig. 4. Most of the additional points lie in the midmorning sector as expected since Cassini was located on the dayside (near noon local time) for the new passes, and the line of sight to the source regions intersects both a dayside and a nightside position, but only the dayside position was
Table 3. Sorting of DF results for Fig. 5.

\begin{tabular}{ccccc}
\hline & $f<300 \mathrm{kHz}$ & $300-600 \mathrm{kHz}$ & $f>600 \mathrm{kHz}$ & Total $(\%)$ \\
\hline$x<x_{o}$ & 1 & 18 & 7 & 5.8 \\
$x_{o}<x<0$ & 41 & 63 & 0 & 23.3 \\
$x>0$ & 150 & 114 & 53 & 70.9 \\
Total $(\%)$ & 43.0 & 43.6 & 13.4 & \\
\hline
\end{tabular}

included in Fig. 5. Very few new points lie on the nightside. Table 3 breaks down the data in Fig. 5, showing similar total percentages to Fig. 4 within each bin, and a slightly larger number falling on the dayside. As we pointed out, for all of these source positions Titan was located on the nightside in the local time range 21:00 $\mathrm{h}<\mathrm{LT}<05: 00 \mathrm{~h}$. Titan's orbit lies at $20.2 R_{\mathrm{S}}$, very near the equatorial plane. Magnetic field lines through Titan will map to high L-shells, perhaps near those of SKR source regions. We comment more on how Titan might influence SKR generation in the Summary and Conclusions section.

\section{Comprehensive survey}

We have recently obtained new processed data results that allow a comprehensive survey of all the 2-antenna mode 


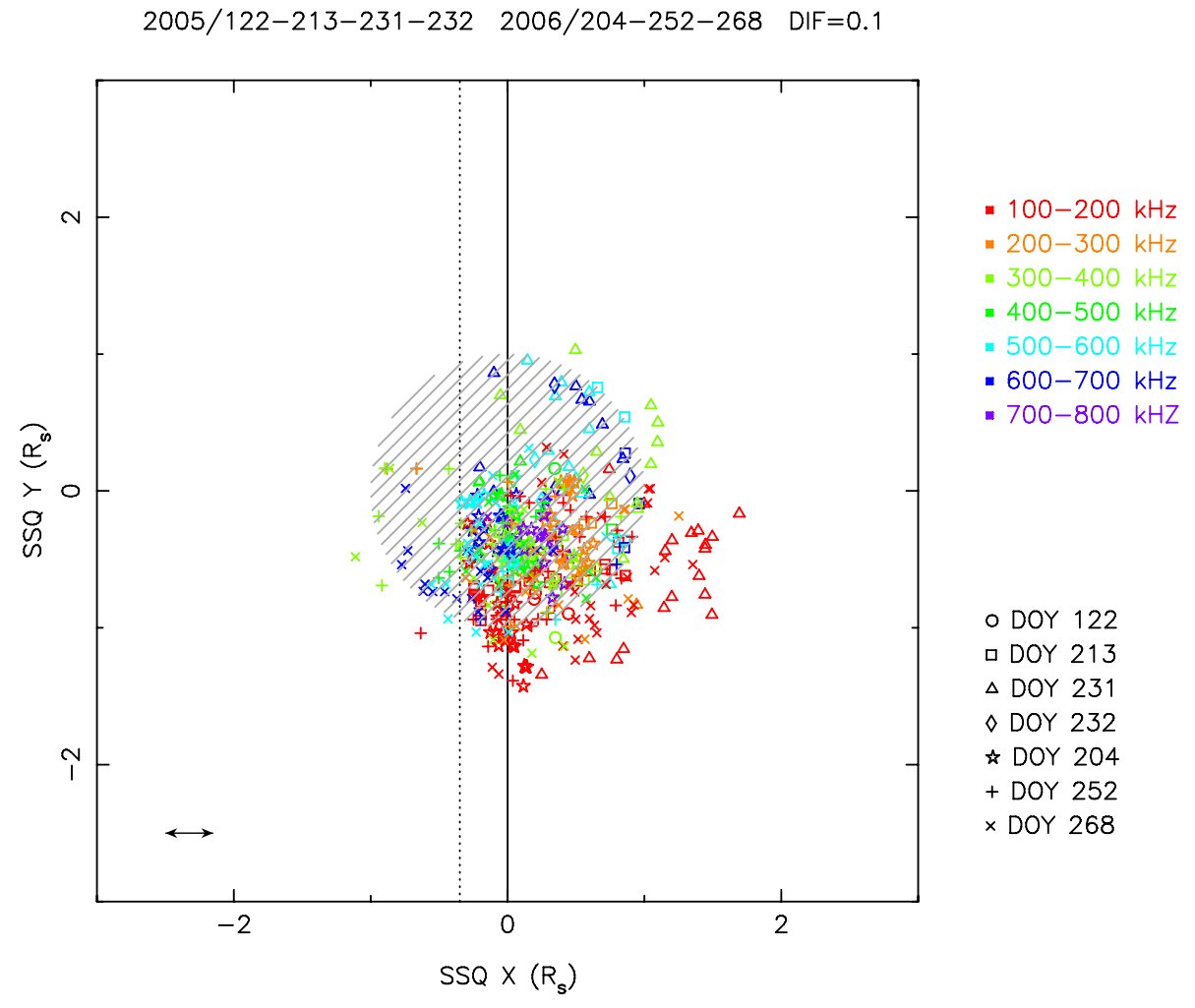

Fig. 5b. Same as Fig. 5a, but now in the $x-y$ plane.

direction-finding results for the mission to date. We have conducted a survey of these data by calculating the location of the SKR source using a technique similar to that used in generating Figs. 4 and 5, but simplified to facilitate a comprehensive search. We have calculated the source location by finding the intersection of the source direction with the surface of cyclotron frequency for the sampled frequency. For a 2-D grid of Titan local time versus SKR source location local time we determine the number of times a source location falls within bins of size $1 \mathrm{~h} \times 1 \mathrm{~h}$. If the direction to the source from the spacecraft does not intersect the cyclotron surface for the sampled frequency, that point is eliminated from the survey. This survey covers the time period from day 170 of 2004 to day 366 of 2008, and frequency range of $100-1000 \mathrm{kHz}$. The data were not constrained to any of the 4 constraints listed earlier for the results shown in Figs. 4 and 5. We show the results in Fig. 6 where the color bar gives the number of points within each bin. This plot shows a clear dominance of SKR LT centered between about 08:00-13:00 h LT. The peaks show more scatter in Titan LT with most points falling in the range 08:00 $\mathrm{h}<\mathrm{LT}_{\text {Titan }}<13: 00 \mathrm{~h}$ with a smaller peak in the range 20:00 $<\mathrm{LT}_{\text {Titan }}<07: 00$. Within this latter range there are two peaks occurring in the ranges 20:00 $<\mathrm{LT}_{\text {Titan }}<23: 00$ and 04:00 $<\mathrm{LT}_{\text {Titan }}<$ 07:00. We find evidence of SKR sources occurring on the nightside of Saturn between 00:00-05:00 h local time.
We have processed the data of Fig. 6 by making a plot of a "cut" of the figure over a limited range of SKR source LT. For each 1-h bin of Titan LT in Fig. 6 we calculate the average of all 1-h bins of SKR source LT over the range 06:00 $\mathrm{h}$ to 14:00 h SKR source LT. In Fig. 7a we show the plot of these averages, normalized to the maximum number of counts. We note peaks in the range of $\mathrm{LT}_{\text {Titan }}$ mentioned above. In Fig. $7 \mathrm{~b}$ we show a similar cut over the range of SKR source LT from 00:00 $\mathrm{h}$ to 05:00 $\mathrm{h}$, including nightside sources only. Here we see the clear peak in nightside sources of SKR occurs when Titan is located 00:00 $<\mathrm{LT}_{\text {Titan }}<05: 00$.

\section{Summary and conclusions}

In this paper we re-examine the occurrence probability of SKR emissions using a much larger database than the original study of Menietti et al. (2007). We have used the new definition of Saturn longitude system based on a variable period as presented in Kurth et al. (2007, 2008). This system organizes the radio emission data and allows us to investigate influence of the occurrence probability of SKR on Saturnian moons. The data are analyzed for frequencies of SKR in the range $100 \mathrm{kHz}<f<900 \mathrm{kHz}$, and for times from 18 June 2004 to 29 June 2006 inclusive.

We find the results agree with the general conclusions of Menietti et al. (2007) as well as Khodachenko et al. (2008). 


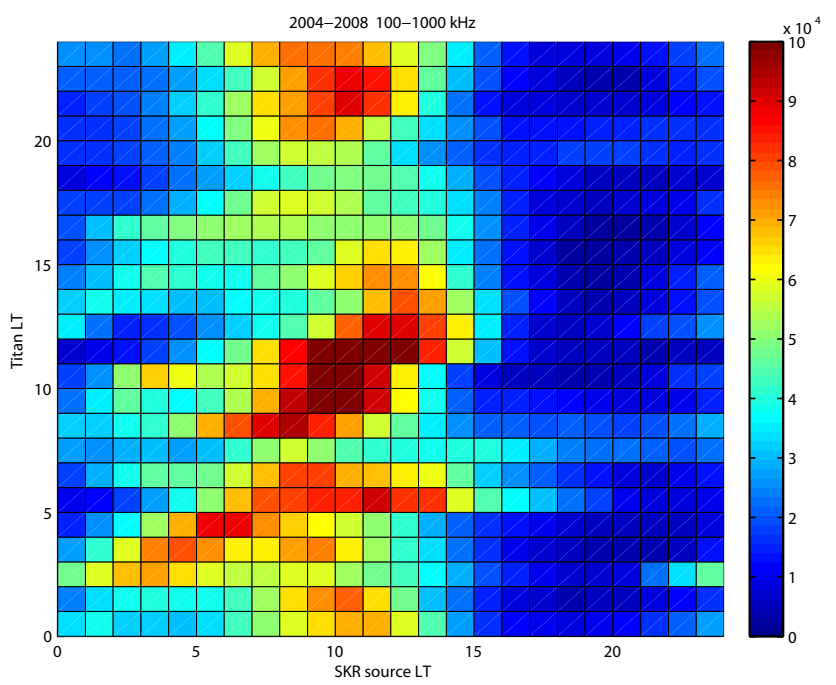

Fig. 6. Titan local time versus local time of SKR source location. The bins size is $1 \mathrm{~h} \times 1 \mathrm{~h}$ and the number of times a source location falls within a bin is color coded. The plot covers the time period from day 170 of 2004 to day 366 of 2008 , and a frequency range of $100-1000 \mathrm{kHz}$.

We isolate emission associated with the orbital position of Titan and determine the direction to the source at those times. Some of this emission does appear to result from field lines that map to the nightside of Saturn.

Previous studies have shown that Titan's local time appears to have an influence on the occurrence probability of Saturn Kilometric Radiation (SKR). Using a more extensive set of data than Menietti et al. (2007), we confirm the correlation of higher occurrence probability of SKR when Titan is located near local midnight. Our results indicate an increase in the occurrence probability of SKR when Titan is located near a local time of midnight $(21: 00 \mathrm{~h}<\mathrm{LT}<05: 00 \mathrm{~h})$ in the Saturn magnetotail. There is a secondary increase in OP of $\mathrm{SKR}$ in the local time range 08:00 $\mathrm{h}<\mathrm{LT}<10: 00 \mathrm{~h}$. This increase may be associated with the location of Cassini during the data sampling. The line of apsides of the Cassini orbit was centered near 08:00-09:00 h of local time for the time period prior to November 2006, before it precessed clockwise to earlier hours of local time. However, there may be another explanation for this increase in OP as discussed below.

As mentioned earlier, SKR is believed to be emitted in a hollow cone with a large wave normal angle. Lamy et al. (2008a, b) and Cecconi et al. (2009) reported on measured beam angles of SKR. The latter authors, for instance observe beaming angles of $50^{\circ}-80^{\circ}$ for $80-400 \mathrm{kHz}$ with some frequency dependence. As discussed by Cecconi et al. (2009) and others radio emission should not be observed for frequencies obscured by the radio horizon, which is approximated by the iso-contours of $f_{\mathrm{c}}$. A radio shadow zone has been reported by Lamy et al. (2008a, b) that obscures ra-

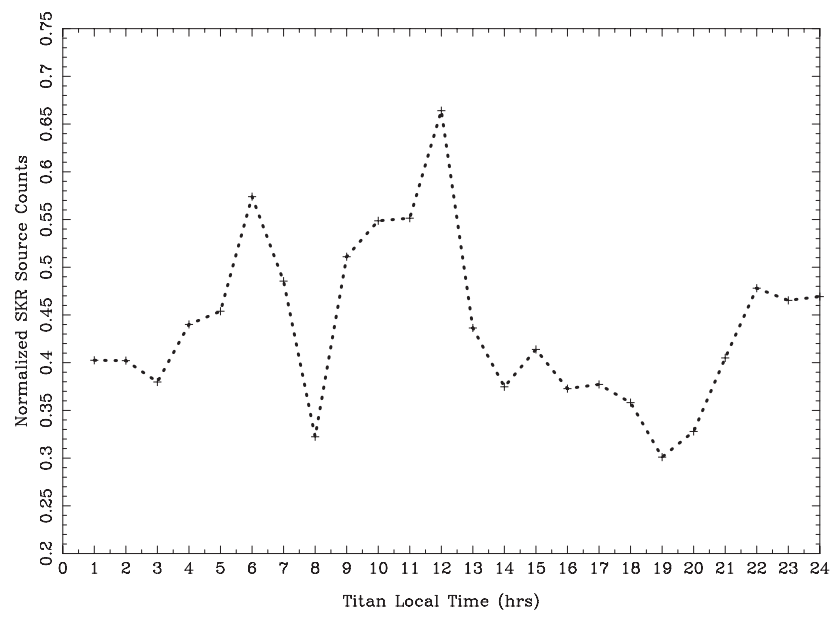

Fig. 7a. A cut or slice of Fig. 6 showing the normalized number of counts versus Titan local time. For each 1-h bin of Titan LT in Fig. 6 we calculate the average of all 1-h bins of SKR source LT over the range $06: 00 \mathrm{~h}$ to $14: 00 \mathrm{~h}$ SKR source LT. The peaks appear as described in the text.

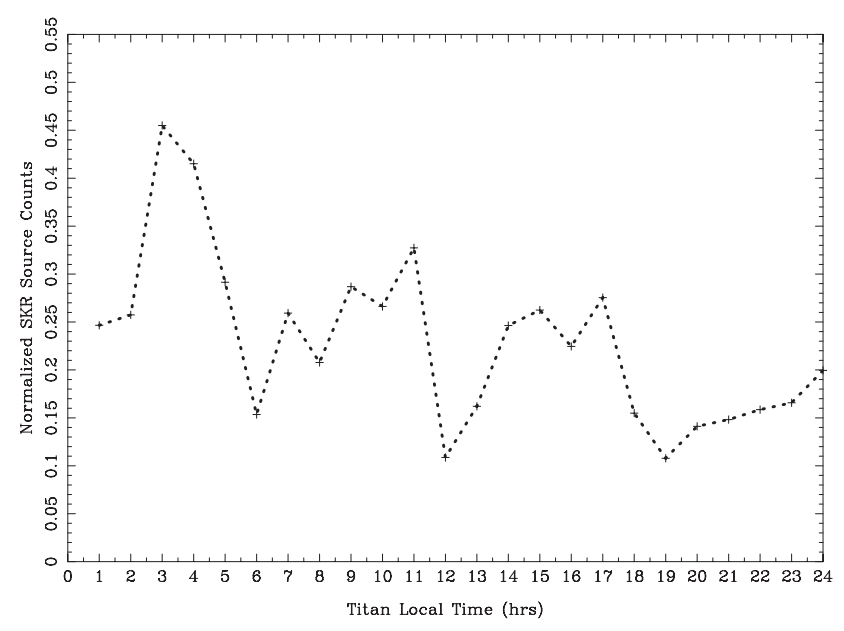

Fig. 7b. The same as Fig. 7a, except that we calculate the average of all 1-h bins of SKR source LT over the range of 00:00 $\mathrm{h}$ to $05: 00 \mathrm{~h}$, thus including nightside sources only. Now the most significant peak occurs for a Titan LT near 03:00 h.

dio emission particularly at high frequencies. Radio sources exist, particularly at low frequency and at locations at large angles away from Cassini that will not be observed. In addition the orbit of Cassini was not always at high latitudes during our survey (as discussed earlier), where it might better observe SKR radio sources.

We have conducted two independent direction finding surveys. In the first, we have applied a set of selection criteria to the dataset to isolate emission that likely results from Titan's location near a local time of midnight. The results, presented in Figs. 4 and 5, indicate that SKR has most source regions located in the mid-morning sector of local time when Titan 
is located near midnight. However, some of the sources of SKR that are associated with times when Titan is near midnight are consistent with nightside source regions, and with electron precipitation from field lines that may have recently mapped to Titan.

The second direction finding survey was conducted with no criteria of data selection, and utilized a much larger database. The survey results, presented in Fig. 6, provide new insight into the location of SKR sources influenced by Titan. There is evidence of nightside source regions within the range of local times 00:00-05:00 h. This emission may be the result of magnetic field lines that have recently reconnected due to Titan's influence near the magnetotail. These field lines then co-rotate producing a trail of nightside source regions, perhaps similar to the footprint regions left by Io, Ganymede, and Europa at Jupiter and observed by the Hubble telescope (cf. Clarke et al., 2002). This concept is described by the theory proposed by Cowley et al. (2005). These authors show how reconnection combined with Saturn rotation yields spiral structures in the same sense as observed in auroral images. In addition, Radioti et al. (2009) have correlated transient auroral spots with energetic particle injections detected by Cassini.

It is most interesting that the most significant peak in the center of Fig. $6\left(08: 00 \mathrm{~h}<\mathrm{LT}_{\text {SKRsource }}<13: 00 \mathrm{~h}\right.$ and 08:00 $\mathrm{h}<\mathrm{LT}_{\text {Titan }}<13: 00 \mathrm{~h}$ ) indicates that Titan may influence even the dominant source of SKR emission reported by Lamy et al. (2008a) not previously associated with Titan. The association of Titan with this SKR source is seen also in Fig. 2 (and in Fig. 4 of Menietti et al., 2007), but was thought previously to be the result of data sampling. It appears the presence of Titan in the mid-morning local time sector may stimulate the generation of SKR.

Titan's presence in the magnetotail may contribute to the efficiency of reconnection and/or may increase the rate of plasma onto reconnected field lines that map to the auroral region and active SKR generation sites. We know that Titan is a significant source of pick-up ions and mass loading (cf. Mitchell et al., 2005; Szego et al., 2005), and is believed to have Alfven wings and an extensive wake (cf. Neubauer et al., 2006; Ma et al., 2006; Modolo et al., 2007; Modolo and Chanteur, 2008). As mentioned above, evidence for Saturn magnetic storms has been shown by Jackman et al. (2007, 2008, 2009) and Russell et al. (2008). More recently, Zaitsev et al. (2010) argue that magnetic field lines that intercept Titan should carry a field-aligned potential of a few kilovolts due to the induced ionospheric electric field resulting from the motion of Titan through the Saturnian magnetosphere. This potential would then accelerate electrons along the Titan flux tube which could be a source of SKR closer to Saturn near the point of gyroresonance via the cyclotron maser mechanism. Such accelerated electrons might be observed near Titan in the future.

As discussed by Winglee et al. (2009), during antiparallel IMF Titan's ion tail interacts with plasma fingers pro- duced by the interchange instability, and can modify the inner boundary of the plasmasheet possibly affecting substorm generation. If the ion tail acts as a barrier to magnetospheric flows, then regions of hot plasma form which might provide a source of precipitating electrons for the generation of SKR. Future multifluid studies of such detailed interactions, perhaps including hybrid simulations, may be valuable to our understanding of how Titan influences the generation of SKR.

Menietti et al. (2007) reported a diminution of SKR when Titan is located in the approximate range $12: 00 \mathrm{~h}<\mathrm{LT}<18: 00 \mathrm{~h}$, and attributed this to a disruption of the normal reconnection process that occurs on the dayside. It was suggested that the Titan plasma wake may somehow inhibit or decrease the efficiency of the reconnection process, perhaps by distorting the location of the magnetopause reconnection site. The diminution of SKR observed in the original study is not as obvious in the study reported here, and what is present seems to be shifted to larger values of local time, perhaps centered near 19:00 h LT as seen in Fig. 2. If the original concept of distortion of the magnetopause by the plasma wake of Titan is correct, that would imply that such distortions were not as common in the extended dataset used for the present study.

Acknowledgements. We thank J. Hospodarsky for clerical assistance and J. B. Groene for critical software development. This work was supported by Jet Propulsion Laboratory Contract 1356500 to the University of Iowa.

Topical Editor R. Nakamura thanks two anonymous referees for their help in evaluating this paper.

\section{References}

Cecconi, B.: Influence of an extended source on Goniopolarimetry (or Direction Finding) with Cassini and STEREO radio receivers, Radio Sci., 42, RS2003, doi:10.1029/2006RS003458, 2007.

Cecconi, B. and Zarka, P.: Direction finding and antenna calibration through analytical inversion of radio measurements performed using a system of two or three electric dipole antennas on a three-axis stabilized spacecraft, Radio Sci., 40, RS3003, doi:10.1029/2004RS003070, 2005.

Cecconi, B., Zarka, P., and Kurth, W. S.: SKR polarization and source localization with the Cassini/RPWS/HFR instrument: First results, in: Planetary Radio Emissions VI, edited by: Rucker, H. O., Kurth, W. S., and Mann, G., pp. 37-49, Austrian Academy of Sciences Press, Vienna, 2006.

Cecconi, B., Lamy, L., Zarka, P., Prangé, R., Kurth, W. S., and Louarn, P.: Goniopolarimetric study of the revolution 29 perikrone using the Cassini Radio and Plasma Wave Science instrument high-frequency radio receiver, J. Geophys. Res., 114, A03215, doi:1029/2008JA013830, 2009.

Clarke, J. T., Ajello, J., Ballester, G., Ben Jaffel, L., Connerney, J., Gérard, J.-C., Gladstone, G. R., Grodent, D., Pryor, W., Trauger, J., and Waite Jr., J. H.: Ultraviolet emissions from the magnetic footprints of Io, Ganymede and Europa on Jupiter, Nature, 415, 997-1000, 2002. 
Cowley, S. W. H., Badman, S. V., Bunce, E. J., Clarke, J. T., Gerard, J.-C., Grodent, D., Jackman, C. M., Milan, S. E., and Yeoman, T. K.: Reconnection in a rotation-dominated magnetosphere and its relation to Saturn's auroral dynamics, J. Geophys. Res., 110, A02201, doi:10.1029/2004JA010796, 2005.

Farrell, W. M., Desch, M. D., Kaiser, M. L., Lecacheux, A., Kurth, W. S., Gurnett, D. A., Cecconi, B., and Zarka, P.: A nightside source of Saturn's kilometric radiation: Evidence for an inner magnetosphere energy driver, Geophys. Res. Lett., 32, L18107, doi:10.1029/2005GL023449, 2005.

Fischer, G., Cecconi, B., Lamy, L., Ye, S., Taubenschuss, U., Macher, W., Zarka, P., Kurth, W. S., and Gurnett, D. A.: Elliptical polarization of Saturn kilometric radiation observed from high latitudes, J. Geophys. Res., 114, A08216, doi:1029/2009JA014176, 2009.

Gurnett, D. A., Kurth, W. S., and Scarf, F. L.: Plasma waves near Saturn: Initial results from Voyager 1, Science, 212, 235-239, 1981.

Gurnett, D. A., Kurth, W. S, Kirchner, D. L., Hospodarsky, G. B., Averkamp, T. F., Zarka, P., Lecacheux, A., Manning, R., Roux, A., Canu, P., Cornilleau-Wehrlin, N., Galopeau, P., Meyer, A., Bostrom, R., Gustafsson, G., Wahlund, J.-E., Ahlen, L., Rucker, H. O., Ladreiter, H. P., Macher, W., Woolliscroft, L. J. C., Alleyne, H., Kaiser, M. L., Desch, M. D., Farrell, W. M., Harvey, C. C., Louarn, P., Kellogg, P. J., Goetz, K., and Pedersen, A.: The Cassini Radio and Plasma Wave Investigation, Space Sci. Rev., 114, 395-463, 2004.

Jackman, C. M., Russell, C. T., Southwood, D. J., Arridge, C. S., Achilleos, N., and Dougherty, M. K.: Strong rapid dipolarizations in Saturn's magnetotail: In situ evidence of reconnection, Geophys. Res. Lett., 34, L11203, doi:10.1029/2007GL029764, 2007.

Jackman, C. M., Arridge, C. S., Krupp, N., Bunce, E. J., Mitchell, O. G., McAndrews, H. J., Dougherty, M. K., Russell, C. T., Achilleos, N., Jones, G. H., and Coates, A. J.: A multiinstrument view of tail reconnection at Saturn, J. Geophys. Res., 113, A11213, doi:10.1029/2008JA013592, 2008.

Jackman, C. M., Lamy, L., Freeman, M. P., Zarka, P., Ceconi, B., Kurth, W. S., Cowley, S. W. H., and Doughery, M. K.: On the character and distribution of lower-frequency radio emissions at Saturn and their relationship to substorm-like events, J. Geophys. Res., 114, A08211, doi:101029/2008JA013997, 2009.

Kaiser, M. L. and Desch, M. D.: Saturnian kilometric radiation: Source locations, J. Geophys. Res., 87, 4555-4559, 1982.

Kaiser, M. L., Desch, M. D., and Lecacheux, A.: Saturnian kilometric radiation: Statistical properties and beam geometry, Nature, 292, 731-733, 1981.

Kaiser, M. L., Desch, M. D., Kurth, W. S., Lecacheux, A., Genova, F., Pedersen, B. M., and Evans, D. R.: Saturn as a radio source, in: Saturn, edited by: Gehrels, T. and Shapley Matthews, M., pp. 378-415, The University of Arizona Press, Tuscon, Arizona, 1984.

Khodachenko, M. L., Panchenko, M. G., Kislyakov, A. G., Taubenschuss, U., and Rucker, H. O.: Possible manifestation of Saturnian moons in the long-periodic variations of SKR, in EPSC Abstracts, 3, pp. EPSC2008-A-00.050, Europlanet, 2008.

Kurth, W. S., Gurnett, D. A., Clarke, J. T., Zarka, P., Desch, M. D., Kaiser, M. L., Cecconi, B., Lecacheux, A., Farrell, W. M., Galopeau, P., Gérard, J.-C., Grodent, D, Prangé, R., Dougherty,
M. K., and Crary, F. J.: An Earth-like correspondence between Saturn's auroral features and radio emission, Nature, 433, 722725, 2005.

Kurth, W. S., Lecacheux, A., Averkamp, T. F., Groene, J. B., and Gurnett, D. A.: A Saturnian longitude system based on a variable kilometric radiation period, Geophys. Res. Lett., 34, L02201, doi:10.1029/2006GL028336, 2007.

Kurth, W. S., Averkamp, T. F., Gurnett, D. A., Groene, J. B., and Lecacheux, A.: An update to a Saturnian longitude system based on kilometric radio emissions, J. Geophys. Res., 113, A05222, doi:10.1029/2007JA012861, 2008.

Lamy, L., Zarka, P., Cecconi, B., Prange, R., Kurth, W. S., and Gurnett, D. A.: Saturn kilometric radiation: Average and statistical properties, J. Geophys. Res., 113, A07201, doi:10.1029/2007JA012900, 2008a.

Lamy, L., Zarka, P., Cecconi, B., Hess, S., and Prange, R.: Modelling of Saturn kilometric radiation arcs and equatorial shadow zone, J. Geophys. Res., 113, A10213, doi:10.1029/2008JA013464, 2008b.

Lamy, L., Cecconi, B., Prange, R., Zarka, P., Nichols, J. D., and Clarke, J. T.: An auroral oval at the footprint of Saturn's radiosources, colocated with the UV aurorae, J. Geophys. Res., 114, A10, doi:10.1029/2009JA014401, 2009.

Lecacheux, A. and Genova, F.: Source location of Saturnian kilometric radio emission, J. Geophys. Res., 88, 8993-8998, 1983.

Louarn, P., Kurth, W. S., Gurnett, D. A., Hospodarsky, G. B., Persoon, A. M., Cecconi, B., Lecacheux, A., Zarka, P., Canu, P., Roux, A., Rucker, H. O., Farrell, W. M., Kaiser, M. L., Andre, N., Harvey, C., and Blanc, M.: Observations of similar radio signatures at Saturn and Jupiter: Implications for the magnetospheric dynamics, Geophys. Res. Lett., 34 , L20113, doi:10.1029/2007GL030368, 2007.

Ma, Y., Nagy, A. F., Cravens, T. E., Sokolov, I. V., Hansen, K. C., Wahlund, J.-E., Crary, F. J., Coates, A. J., and Dougherty, M. J.: Comparisons between MHD model calculations and observations of Cassini flybys of Titan, J. Geophys. Res., 111, A05207, doi:10.1029/2005JA011481, 2006.

Menietti, J. D., Groene, J. B., Averkamp, T. F., Hospodarsky, G. B., Kurth, W. S., Gurnett, D. A., and Zarka, P.: Influence of Saturnian moons on Saturn kilometric radiation, J. Geophys. Res., 112, A08211, doi:10.1029/2007JA012331, 2007.

Menietti, J. D., Ye,S.-Y., Yoon, P. H., Santolik, O., Rymer, A. M., Gurnett, D. A., and Coates, A. J.: Analysis of narrowband emission observed in the Saturn magnetosphere, J. Geophys. Res., 114, A06206, doi:10.1029/2008JA013982, 2009.

Mitchell, D. G., Brandt, P. C., Roelof, E. C., Dandouras, J., Krimigis, S. M., Mauk, B. H., Paranicas, C. P., Krupp, N., Hamilton, D. C., Kurth, W. S., Zarka, P., Dougherty, M. K., Bunce, E. J., Shemansky, D. E.: Energetic ion acceleration in Saturn's magnetotail: Substorms at Saturn?, Geophys. Res. Lett., 32, L20S01, doi:10.1029/2005GL022647, 2005.

Modolo, R. and Chanteur, G. M.: A global hybrid model for Titan's interaction with the Kronian plasma: Application to the Cassini Ta flyby, J. Geophys. Res., 113, A01317, doi:10.1029/2007JA012453, 2008.

Modolo, R., Chanteur, G. M., Wahlund, J.-E., Canu, P., Kurth, W. S., Gurnett, D., Matthews, A. P., and Bertucci, C.: Plasma environment in the wake of Titan from hybrid simulation: A case study, Geophys. Res. Lett., 34, L24S07, 
doi:10.1029/2007GL030489, 2007.

Neubauer, F. M., Backes, H., Dougherty, M. D., Wennmacher, A., Russell, C. T., Coates, A., Young, D., Achilleos, N., Andre, N., Arridge, C. S., Bertucci, C., Jones, G. H., Khurana, K. K., Knetter, T., Law, A., Lewis, G. R., and Saur, J.: Titan's near magnetotail from magnetic field and electron plasma observations and modeling: Cassini flybys TA, TB, and T3, J. Geophys. Res., 111, A10220, doi:10.1029/2006JA011676, 2006.

Porco, C. C., Helfenstein, P., Thomas, P. C., et al.: Cassini observes the active south pole of Enceladus, Science, 311(5766), 13931401, 2006.

Radioti, A., D. Grodent, J., Gerard, C., Roussos, E., Paranicas, C., Bonfond, B., Mitchell, D. G., Krupp, N., Krimigis, S., and Clarke, J. T.: Transient auroral features at Saturn: Signatures of energetic particle injections in the magnetosphere, J. Geophys. Res., 114, A03210, doi:10.1029/2008JA013632, 2009.

Russell, C. T., Jackman, C. M., Wei, H. Y., Bertucci, C., and Dougherty, M. K.: Titan's influence on Saturnian substorm occurrence, Geophys. Res. Lett., 35, L12105, doi:10.1029/2008GL034080, 2008.

Szego, K., Bebesi, Z., Erdos, G., Foldy, L., Crary, F., McComas, D. J., Young, D. T., Bolton, S., Coates, A. J., Rymer, A. M., Hartle, R. E., Sittler, E. C., Reisenfeld, D., Bethelier, J. J., Johnson, R. E., Smith, H. T., Hill, T. W., Vilppola, J., Steinberg, J., and Andre, N.: The global plasma environment of Titan as observed by Cassini Plasma Spectrometer during the first two close encounters with Titan, Geophys. Res. Lett., 32, L20S05, doi:10.1029/2005GL022646, 2005.
Wang, Z., Gurnett, D. A., Kurth, W. S., Ye, S.-Y., Mitchell, D. G., Carbary, J. F., and Russell, C. T.: Narrowband radio emissions and their relationships to rotating plasma clouds and magnetic disturbances at Saturn, European Geophysical Union General Assembly, Vienna, Austria, 13-18 April, Abstract, PS8-ITU50003, 2008.

Warwick, J. W., Pearce, J. B., Evans, D. R., Carr, T. D., Schauble, J. J., Alexander, J. K., Kaiser, M. L., Desch, M. D., Pedersen, B. M., Lecacheux, A., Daigne, G., Boischot, A., and Barrow, C. H.: Planetary radio astronomy observations from Voyager 1 near Saturn, Science, 212, 239-243, 1981.

Warwick, J. W., Evans, D. R., Romig, J. H., Alexander, J. K., Desch, M. D., Kaiser, M. L., Aubier, M., Leblanc, Y., Lecacheux, A., and Pedersen, B. M.: Planetary radio astronomy observations from Voyager 2 near Saturn, Science, 215, 582-587, 1982.

Winglee, R. M., Snowden, D., and Kidder, A.: Modification of Titan's ion tail and the Kronian magnetosphere: Coupled magnetospheric simulations, J. Geophys. Res., 114, A05215, doi:10.1029/2008JA013343, 2009.

Ye, S.-Y., Gurnett, D. A., Fischer, G., Cecconi, B., Menietti, J. D., Kurth, W. S., Wang, Z., Hospodarsky, G. B., Zarka, P., and Lecaceux, A.: Source locations of narrowband radio emissions detected at Saturn, J. Geophys. Res., 114, A06219, doi:10.1029/2008JA013855, 2009.

Zaitsev, V. V., Shaposhnikov, V. E., Khodachenko, M. L., Rucker, H. O., and Panchenko, M.: Acceleration of electrons in Titan's ionosphere, J. Geophys. Res., in press, doi:10.1029/2008JA013958, 2010.

Zarka, P.: Auroral radio emissions at the outer planets: Observations and theories, J. Geophys. Res., 103, 20159-20194, 1998. 